\title{
Validity of the PARADISE24 questionnaire in people with substance use disorders : A measure to assess psychosocial difficulties
}

\section{de la Fuentes, Javier}

2018-06-01

de la Fuentes , J , Cabello, M , Levola , J, Felix Caballero , F , Luis Ayuso-Mateos , J \&

Pitkänen , T 2018, ' Validity of the PARADISE24 questionnaire in people with substance use disorders : A measure to assess psychosocial difficulties ', Drug and Alcohol Dependence , vol. 187 , pp. 66-71 . https://doi.org/10.1016/j.drugalcdep.2018.02.012

http://hdl.handle.net/10138/303669

https://doi.org/10.1016/j.drugalcdep.2018.02.012

publishedVersion

Downloaded from Helda, University of Helsinki institutional repository.

This is an electronic reprint of the original article.

This reprint may differ from the original in pagination and typographic detail.

Please cite the original version. 


\title{
Validity of the PARADISE24 questionnaire in people with substance use disorders: A measure to assess psychosocial difficulties
}

\author{
Javier de la Fuente ${ }^{\mathrm{a}}$, Maria Cabello ${ }^{\mathrm{a}, \mathrm{b}, \mathrm{c}, *}$, Jonna Levola ${ }^{\mathrm{d}}$, Francisco Félix Caballero ${ }^{\mathrm{a}, \mathrm{b}, \mathrm{c}}$, \\ José Luis Ayuso-Mateos ${ }^{\mathrm{a}, \mathrm{b}, \mathrm{c}}$, Tuuli Pitkänen ${ }^{\mathrm{e}}$ \\ a Department of Psychiatry, School of Medicine, Universidad Autónoma de Madrid, Ciudad Universitaria de Cantoblanco, 28049, Madrid, Spain \\ b Centro de Investigation Biomédica en Red de Salud Mental, Cibersam, C/Diego de León, 62, 28006, Madrid, Spain \\ ${ }^{\mathrm{c}}$ Department of Psychiatry, Hospital Universitario de La Princesa, Instituto de Investigación Sanitaria Princesa (IIS Princesa), C/Diego de León, 62, 28006, Madrid, Spain \\ d Department of Psychiatry, Hyvinkää Hospital Area, Hospital District of Helsinki and Uusimaa, Sairaalankatu 1, 05850, Hyvinkää, Finland \\ e A-Clinic Foundation, Ratamestarinkatu 7 A, 00520, Helsinki, Finland
}

A R T I C L E I N F O

\section{Keywords:}

PARADISE24

Substance use disorders

Validation

Factor analysis

Alcohol

\begin{abstract}
A B S T R A C T
Objectives: Psychosocial difficulties (PSDs) are common in people with substance use disorders (SUDs). The PARADISE24 has been shown to be an adequate tool for measuring PSDs in inpatients with SUDs. The aim of this study is to evaluate the psychometric properties of the PARADISE24 in a sample of patients with SUDs. Methods: 2637 participants with SUDs completed the PARADISE24 questionnaire during their treatment. The latent structure of the PARADISE24 questionnaire was analyzed in the outpatient sample by means of exploratory and confirmatory factor analysis (EFA and CFA). Metric invariance was then assessed in relation to the inpatient sample using multiple group CFA. Finally, evidences of known-groups validity were checked to test the ability of the questionnaire to differentiate between socio-demographic and clinical groups.

Results: The one-factor model presented an adequate fit in both the EFA (CFI $=0.98$; TLI $=0.98$; RMSEA $=0.07)$ and the CFA (CFI $=0.98$; TLI $=0.98$; RMSEA $=0.07$ ) solutions. The reliability of the scale was found to be high $(\alpha=0.93)$. Strict metric invariance between inpatients and outpatients was achieved $($ RMSEA $=0.063$; TLI $=0.983$; CFI $=0.981)$. The PARADISE24 was able to discriminate between the inpatients and outpatients at both latent $(d=0.98)$ and observed levels $(d=0.86)$.

Conclusions: The PARADISE24 is a unidimensional tool that is reliable for assessing and comparing PSDs in both outpatients and inpatients with SUDs. Further research is required for evaluating the ability of the PARADISE24 to quantify longitudinal changes in PSDs.
\end{abstract}

\section{Introduction}

Drug and alcohol use disorders are common in many European countries and are associated with problems and expenses to society (Organisation for Economic Co-operation and Development, 2014). Although there is a considerable treatment gap in people with substance use disorders (SUDs), it is estimated that $90 \%$ of people with drug dependence and 54\% of people with alcohol dependence seek help at some point of their life (Blanco et al., 2015).

Validated and reliable measures are needed for the evaluation of needs and treatment effects. Traditionally, the effectiveness of SUD treatment has been measured by the duration of abstinence, number of relapses, or addiction severity scores (Black et al., 2017; Dutra et al., 2008). However, alongside harm reduction and withdrawal management, enhancing psychosocial functioning has been recognized as an important goal of SUD treatment (Department of Health, 2007; Moyer, 2013; World Health Organization, 2016). Psychosocial difficulties (PSDs) including emotional, cognitive, and social problems affect everyday life. PSDs are widely experienced in people with substance use disorders (Levola et al., 2014; Poudel et al., 2016). In addition, some PSDs have been reported to be related to subsequent relapses (Moore et al., 2014) and poor treatment outcomes in SUDs (Stevens et al., 2014). It has therefore become increasingly necessary to incorporate information on PSDs in clinical practice in order to effectively personalize treatment of SUDs and to monitor treatment progress and prevent relapses (Luquiens et al., 2012).

The concept of PSDs is not uniform in the literature and includes different areas ranging from problems in daily functioning to mental health disorders (Vannieuwenborg et al., 2015). According to the International Classification of Functioning, Disability and Health (ICF),

\footnotetext{
* Corresponding author at: Hospital Universitario de la Princesa, Cibersam, C/Diego de León, 62, 28006, Madrid, Spain.

E-mail address: maria.cabello@uam.es (M. Cabello).
} 
disability is more than the mere presence of health conditions (World Health Organization, 2001). PSDs have been operationalized in the ICF framework as "the impairments in mental and body functions under nervous-system control, activity limitations and participation restrictions that result from the interaction of a person with a brain disorder and the environmental and personal factors" (Cieza et al., 2015a). Therefore, PSDs are not mental health disorders but may be the consequences of mental health disorders.

The PARADISE24 instrument was created in a multi-country effort to measure common psychosocial difficulties in mental and neurological disorders. The instrument showed adequate properties in a sample of nine different health conditions, including inpatients with SUDs (Cieza et al., 2015b). However, there is still no evidence of the PARADISE24 latent structure in a sample of outpatients with SUDs examining whether the structure of the questionnaire is the same between inpatient and outpatient groups with SUDs.

The aim of this study was to evaluate the psychometric properties of the PARADISE24 with five response options in a sample of outpatients with SUDs, focusing on i) the latent structure of the questionnaire, ii) the metric invariance in relation to a sample of inpatients with SUD, and iii) its ability to discriminate across different demographic and clinical groups.

\section{Methods}

\subsection{Design and sample}

The present work is a psychometric study based on cross-sectional registry data which included a convenience sample of 2851 participants with SUDs (alcohol 57.5\%, opioids 17.8\%, stimulants 8.0\%, cannabis $7.0 \%$ and sedatives $5.5 \%$ coded as primary substance) who completed the PARADISE24 questionnaire during their treatment at the A-Clinic Foundation (Finland). This institution serves around 10,000 outpatients and 5000 inpatients annually. The A-Clinic Foundation has clinics in several large cities in Finland; additionally, Järvenpää Addiction Hospital (JAH) provides inpatient services for SUDs nationally. Data were collected in 2014-2017. The present data included 1376 inpatients at 14 treatment units, $30.0 \%$ of which were at JAH. Most of the studied inpatients $(56.8 \%)$ had completed the questionnaire during detoxification, and the rest (43.2\%) completed the questionnaire during rehabilitation. The data also included 1261 outpatients from 26 treatment units. Outpatient treatment consists largely of therapy and counselling for individuals or groups; the recovery process involves assessing and monitoring patients' health and medication and developing a plan for rehabilitation and follow-up. One fourth $(24.7 \%)$ of the studied outpatients had filled in the questionnaire in the context of opioid substitution treatment, $7.6 \%$ in connection to housing services for substance abusers, $5.4 \%$ at one of the youth clinics that aim to help young people with SUDs, and the rest (62.4\%) at clinics for adults with SUDs. Out of the 2851 patients who had filled in the PARADISE24 questionnaire, 92.5\% (2637) had answered all questions; this was the sample considered in the present study. The use of anonymized register data was approved by the A-Clinic Foundation's Ethical Committee for Treatment and Research and the National Institute for Health and Welfare.

\subsection{Variables}

Participants were asked to fill out the PARADISE24 questionnaire with five response options (PARADISE24fin, 2018) during treatment, and the answers were coded by the health care worker into the electronic treatment record. The questionnaire comprises 24 items evaluating the presence and severity of PSDs in the last 30 days (Cieza et al., 2015b). The items included questions such as: "How much difficulty did you have in remembering to do important things?". Each question was scored on a scale ranging from 0 (no difficulty) to 4 (extreme difficulty/ cannot do). The items were added into an overall mean score to summarize the presence and severity of PSDs (Pitkänen et al., 2016), in which a higher score means higher number and/or severity of PSDs.

Age in years, gender, level of education (coded as less than primary education, primary education completed, secondary education completed, and tertiary education), type of substance used (alcohol use only vs. drug or polysubstance use), and clinical setting (inpatients and outpatients) were collected from the electronic treatment record system. By definition, outpatients visited the clinic for counselling or treatment consisting of several of appointments, whereas inpatients stayed at the clinic overnight.

\subsection{Data analysis}

A general profile comprising socio-demographic and clinical characteristics of the inpatients and outpatients was obtained. Statistical differences between inpatients and outpatients were tested using chisquare tests for categorical variables and two sample $t$-tests for continuous variables. Effect sizes were also computed for each contrast using Cramer's $V$ for the chi-square tests and Cohen's $d$ for the $t$-tests. Cohen's guidelines (Cohen, 1988) were used for interpreting the effect sizes. For Cramer's V, 0.10 represents a small effect size, 0.30 is a medium effect size, and 0.50 is a large effect size. For Cohen's $d, 0.20$, 0.50 , and 0.80 represent, respectively, a small, a medium, and a large effect size.

Subsequently, the latent structure of the PARADISE24 questionnaire was analyzed in the outpatient sample following a two-step factor analysis approach. This sample was randomly divided into two groups: 1) a developmental exploratory subsample, comprising $70 \%$ of the total outpatient's sample, and 2) a validation subsample with the remaining $30 \%$ of this sample. This two-step validation procedure has been implemented in previous studies (Caballero et al., 2017).

An exploratory factor analysis (EFA) was conducted over the estimated polychoric correlations matrix in the developmental subsample. The estimation method used was Weighted Least Squares with mean adjusted statistics (WLSM), and the 'oblimin' rotation was used to allow for potential correlated factors. The number of factors to be selected was based on parallel analysis (PA) (Horn, 1965) and Velicer's minimum average partial (MAP) test (Velicer, 1976).

Secondly, the factor structure identified in the EFA solution was fitted on the outpatient validation sample by means of Confirmatory Factor Analysis (CFA). The fit of the model was assessed considering the Comparative Fit Index (CFI), the Tucker-Lewis Index (TLI), and the Root Mean Square Error of Approximation (RMSEA). Standards proposed by Hu and Bentler (1999) were considered as indicators of an acceptable fit: CFI $>0.90$; TLI $>0.90$; RMSEA $<0.08$. The parameters of the CFA model were estimated over the polychoric correlation matrix using Weighted Least Squares with mean adjusted test statistics (WLSM). Mean adjusted chi-square test statistic with the Satorra-Bentler correction and robust standard errors were implemented for both the CFA and the subsequent metric invariance analysis. The reliability of the scale in the overall outpatient sample was also estimated using the Cronbach's alpha coefficient, which ranges from 0 (no reliability) to 1 (perfect reliability).

Thirdly, measurement invariance between the outpatient and inpatient groups was conducted through multiple group CFA. Metric invariance analysis consisted of a series of nested models with increasing parametric constraint imposition, and it allows for assessing whether the structure, meaning, and metric of the measured construct (i.e., PSDs) are the same for both populations (Gregorich, 2006).

The levels of constraints imposed in each invariance model correspond to 1) configural invariance (factor structure is equal across groups), 2) metric invariance (equal factor loadings), 3) scalar invariance (equal loadings and intercepts), and 4) strict invariance (residual variances are also fixed to be equal across groups). Theta parameterization was used. The measurement invariance analysis was based 
on changes in the CFI values lower than 0.01 across the different nested models (Cheung and Rensvold, 2002). When scalar invariance was achieved, latent mean scores on the general factor were compared between the outpatient and inpatient groups.

Finally, evidences of known-groups validity (Davidson, 2014) were checked by testing mean differences between relevant groups based on socio-demographic variables (i.e., gender, age groups and educational level) and clinical characteristics, such as clinical setting (outpatient vs. inpatients) and type of substance used (alcohol use only vs. drug use problems) in the overall sample. Cohen's $f$ was used as an effect size measure when testing differences across more than 2 groups; $0.1,0.25$, and 0.40 values represent small, medium and large effect sizes, respectively.

PA and Velicer's MAP test were implemented using the $R$ package psych (Revelle, 2008). EFA was carried out in Mplus version 7 (Muthén and Muthén, 2011), while CFA and metric invariance analysis were conducted using the lavaan package in $R$ (Rossel, 2012).

\section{Results}

\subsection{General description of the sample}

Altogether 2637 patients in treatment for substance use disorders filled out the PARADISE24 questionnaire. As can be seen in Table 1, significant differences (although with small effect sizes) were found for gender $(p=0.007$; Cramer's $V=0.05)$, age $(p=0.033$; Cohen's $d=0.08)$, education $(p<0.001$; Cramer's $V=0.11)$, and type of substance used ( $p=0.039$; Cramer's $V=0.07$ ) between the outpatients $(n=1261)$ and inpatients $(n=1376)$. The inpatient group comprised a higher proportion of males.

\subsection{Exploratory factor analysis}

The PA analysis and the Velicer's MAP test implemented in the developmental sample $(n=935)$ indicated that the optimum number of factors to retain was 1 . Results from the one-factor EFA solution presented an adequate fit (CFI $=0.98$; TLI $=0.98$; RMSEA $=0.07$ ), with the factor explaining $45 \%$ of the items' total variance. The standardized estimated factor loadings of the solution (pattern matrix) were all statistically significant $(p<0.001)$ and ranged from 0.41 to 0.80 .

\subsection{Confirmatory factor analysis}

Considering the results from the previous EFA solution, a unidimensional model was fitted in the outpatients' validation sample

Table 1

General profile of the outpatients and inpatients' groups.

\begin{tabular}{|c|c|c|c|c|}
\hline & $\begin{array}{l}\text { Outpatients } \\
n=1261\end{array}$ & $\begin{array}{l}\text { Inpatients } \\
n=1376\end{array}$ & $p$ & Effect size \\
\hline Male, n (\%) & $832(65.98)$ & $975(70.86)$ & 0.007 & 0.05 \\
\hline Age, Mean (S.D.) & $41.12(14.16)$ & 39.99 (12.97) & 0.033 & 0.08 \\
\hline Education & & & $<0.001$ & 0.11 \\
\hline None, n (\%) & $30(2.38)$ & $25(1.82)$ & & \\
\hline $\begin{array}{l}\text { Primary education, } \mathrm{n} \\
\quad(\%)\end{array}$ & $460(36.48)$ & $511(37.14)$ & & \\
\hline $\begin{array}{l}\text { Secondary education, } \\
\mathrm{n}(\%)\end{array}$ & $541(42.90)$ & $541(39.32)$ & & \\
\hline $\begin{array}{l}\text { Tertiary education, } \mathrm{n} \\
\text { (\%) }\end{array}$ & $134(10.63)$ & $111(8.07)$ & & \\
\hline Type of substance & & & 0.039 & 0.07 \\
\hline Only alcohol, n (\%) & $585(50.56)$ & $572(49.44)$ & & \\
\hline $\begin{array}{l}\text { Drugs or multiple } \\
\text { substances, n (\%) }\end{array}$ & $601(43.14)$ & $792(56.86)$ & & \\
\hline
\end{tabular}

Note: Cramer's $V$ was used as effect size measure in the comparisons between categorical variables, while Cohen's $d$ was used as effect size measure in the comparisons between continuous variables.
( $n=437)$. Results from the CFA revealed an adequate fit of the unidimensional model in the outpatient's validation sample $(\mathrm{CFI}=0.98$; $\mathrm{TLI}=0.98$; RMSEA $=0.07$ ). Standardized factor loadings of the unidimensional solution (Table 2) were all statistically significant $(p<0.001)$ and ranged from 0.43 to 0.80 .

\subsection{Reliability of the item scores}

The reliability of the scale was assessed in the overall outpatient sample before conducting the measurement invariance analysis, and it obtained a high Cronbach's alpha value $(\alpha=0.93)$. The mean interitem correlation was 0.36 , and dropping any item of the scale did not increase the Cronbach's alpha coefficient.

\subsection{Measurement invariance analysis}

Results from the multiple group CFA measurement invariance are presented in Table 3. Models 1-4 presented values of RMSEA lower than 0.05 and values of CFI and TLI higher than 0.95 . No significant increments in CFI $(\triangle \mathrm{CFI}<0.01$ in all cases) were observed in the sequential comparisons conducted over these nested models. The strict invariance (equal residuals) model presented a good fit (RMSEA $=0.063$; TLI $=0.983$; CFI $=0.981$ ). Standardized loadings of the strict invariance model are presented in Table 2 for both outpatient and inpatient groups. Latent mean scores on the general factor were calculated based on the scalar invariance model, and significant differences $(p<0.001)$ were found between the two groups with higher scores in the inpatient group $(d=0.98)$.

\subsection{Known-groups validity}

As shown in Table 4, statistically significant differences in the PARADISE24 overall mean scores were found across all background variables. The total mean score was higher (more frequent and/or severe PSDs) for females than for males $(d=0.11)$ and for the less educated in comparison with those who had completed at least secondary education $(d=0.15)$. Younger patients had slightly higher PARADISE24 mean scores than older patients $(f=0.14)$. Regarding clinical variables, people with alcohol use only obtained lower mean scores than those with drug or multiple substance use $(d=0.36)$. Large differences were found in the PARADISE24 overall mean score between outpatients and inpatients $(d=0.86)$, with inpatients showing higher scores.

\section{Discussion}

This article provides evidence regarding the unidimensional structure of the PARADISE24 questionnaire in a sample of Finnish outpatients with SUDs. Unidimensional measures with high internal consistency are essential in psychological assessment, since constructirrelevant variance is one of the most important sources of invalidity in tests and questionnaires (Messick, 1995). In regard to unidimensionality, a strong general factor was found in both the developmental (EFA) and validation (CFA) samples of outpatients with SUDs. The unidimensional model presented adequate goodness-of-fit indices; all items had significant loadings over 0.40 . In terms of reliability, the questionnaire presented high reliability in the overall outpatient sample and had a Cronbach's alpha of 0.93 , indicating a strong internal consistency. According to the unidimensional structure of the PARADISE24, it is possible to calculate an overall total score based on the sum of all item scores. This unidimensional structure has been previously reported in people with different health conditions, including inpatients with SUDs (Cieza et al., 2015b). In addition to stopping or reducing substance use and the harm associated therewith, the aims of SUD treatment include enhancing psychosocial functioning and improving the quality of life of the patients. Our results suggest that the 
Table 2

Standardized factor loadings of the unidimensional model.

Strict metric invariance

\begin{tabular}{|c|c|c|c|}
\hline & $\begin{array}{l}\text { Outpatients' validation } \\
\text { sample }\end{array}$ & Outpatients & Inpatients \\
\hline $\begin{array}{l}\text { 1. How much of a problem did you have due to not feeling rested and refreshed during the day (e.g. feeling tired, not } \\
\text { having energy)? }\end{array}$ & 0.71 & 0.72 & 0.68 \\
\hline 2. How much of a problem did you have with loss of interest? & 0.75 & 0.71 & 0.67 \\
\hline 3. How much of a problem did you have with your appetite? & 0.59 & 0.61 & 0.56 \\
\hline $\begin{array}{l}\text { 4. How much of a problem did you have with sleeping, such as falling asleep, waking up frequently during the night } \\
\text { or waking up too early in the morning? }\end{array}$ & 0.57 & 0.57 & 0.52 \\
\hline $\begin{array}{l}\text { 5. How much of a problem did you have being so irritable that you started arguments, shouted at people or even hit } \\
\text { people? }\end{array}$ & 0.67 & 0.58 & 0.53 \\
\hline $\begin{array}{l}\text { 6. How much of a problem did you have with being slowed down or feeling as if things were moving too fast around } \\
\text { you? }\end{array}$ & 0.69 & 0.68 & 0.63 \\
\hline 7. How much of a problem did you have with feeling sad, low or depressed? & 0.80 & 0.80 & 0.76 \\
\hline 8. How much of a problem did you have with worry or anxiety? & 0.79 & 0.81 & 0.77 \\
\hline 9. How much of a problem did you have with not being able to cope with all the things that you had to do? & 0.78 & 0.79 & 0.75 \\
\hline 10. How much bodily ache or pain did you have? & 0.48 & 0.47 & 0.42 \\
\hline 11. How much difficulty did you have in concentrating on doing something for ten minutes? & 0.73 & 0.72 & 0.67 \\
\hline 12. How much difficulty did you have in remembering to do important things? & 0.69 & 0.71 & 0.67 \\
\hline 13. How much difficulty did you have in making decisions? & 0.74 & 0.75 & 0.71 \\
\hline 14. How much difficulty did you have in starting and maintaining a conversation? & 0.68 & 0.62 & 0.57 \\
\hline 15. How much difficulty did you have in walking a long distance such as a kilometer (or equivalent)? & 0.43 & 0.51 & 0.46 \\
\hline 16. How much difficulty did you have in grooming or dressing, toileting or eating? & 0.68 & 0.69 & 0.64 \\
\hline 17. How much difficulty did you have in sexual activities? & 0.55 & 0.56 & 0.51 \\
\hline 18. How much difficulty did you have in staying by yourself for a few days? & 0.55 & 0.59 & 0.54 \\
\hline $\begin{array}{l}\text { 19. How much difficulty did you have with looking after your health, such as eating well, exercising and taking your } \\
\text { medicines? }\end{array}$ & 0.74 & 0.77 & 0.73 \\
\hline 20. How much difficulty did you have in initiating and maintaining a friendship? & 0.69 & 0.69 & 0.64 \\
\hline 21. How much difficulty did you have in getting along with people who are close to you? & 0.61 & 0.64 & 0.59 \\
\hline 22. How much difficulty did you have in your day-to-day work or school? & 0.64 & 0.66 & 0.61 \\
\hline 23. How much difficulty did you have with managing your money? & 0.55 & 0.63 & 0.58 \\
\hline $\begin{array}{l}\text { 24. How much difficulty did you have in joining in community activities (for example, festivities, religious or other } \\
\text { activities) in the same way as anyone else can? }\end{array}$ & 0.72 & 0.72 & 0.68 \\
\hline
\end{tabular}

Note: In the strict invariance model, the standardized factor loadings are not the same for the inpatients and outpatient's groups due to differences in the latent factor variance.

Table 3

Goodness-of-fit indices for the invariance models.

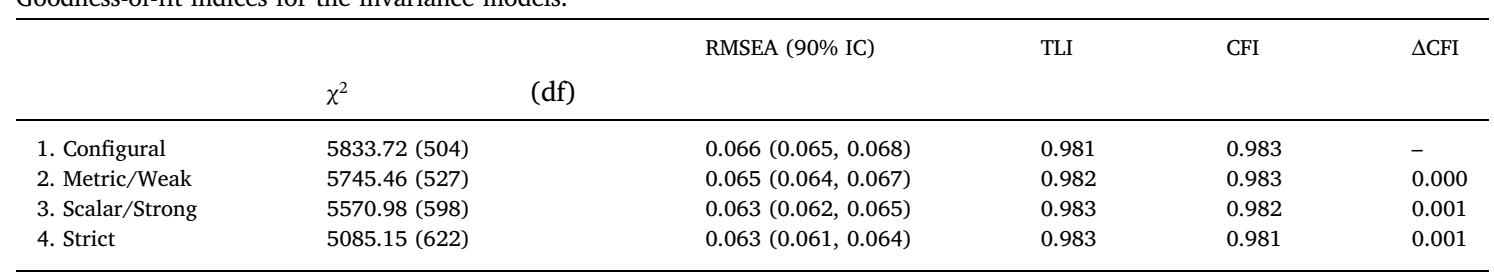

PARADISE24 seems suitable for measuring a wide range of common psychosocial difficulties in SUDs.

Routine assessment of problems related to psychosocial functioning may facilitate communication between health professionals and patients with SUDs in a common and understandable language (Stucki, 2005). Other instruments specific to SUDs, such as the European Addiction Severity Index (EuropASI) (Gsellhofer et al., 1995) or the Alcohol and Drug Outcome Measure (Deering et al., 2009; McLella et al., 1980), include information on some PSDs. However, this information is often incorporated in measures with a broader focus, e.g., assessment of diagnostic criteria or assessment of the severity of dependence. The PARADISE24 also covers a range of issues (for example, psychomotor or self-control problems) that are not usually included in the generic instruments such as the WHO's Disability Assessment Schedule (WHODAS II) (Üstün, 2010), and the 36-item Short Form Survey (Ware, 2000). The application of the PARADISE24 is also probably faster and easier to score than other battery of instruments such as the Measurements in the Addictions for Triage and Evaluation (MATE) (Schippers et al., 2010).

On the other hand, the use of the PARADISE24 has provided a more accurate prospection of total health care costs than the use of symptom- related information in depression and anxiety disorders (Twomey et al., 2017). Further longitudinal studies should verify the clinical utility of the PARADISE24 for individualized treatment planning and prospection of health care resources.

Moreover, our study provides evidence of strict metric invariance between outpatients and inpatients, supporting the use of this tool for assessing and comparing the level of psychosocial difficulties in both inpatient and outpatient populations with SUDs. Useful tools are needed across treatment phases. Our results suggest that PARADISE24 is an adequate instrument to monitor the clinical progress of people with SUDs across treatment phases. The use of the same instrument during different phases of treatment can help to bridge the existing information gap between hospital registries and outpatient health care records (García Álvarez et al., 2011).

The present study also analysed whether the PARADISE24 was able to discriminate between relevant demographic and clinical groups. Results were all congruent with previous literature. Females and younger people reported significantly more frequent and/or severe PSDs problems than males (Garg et al., 1999) and older people (Whiteford et al., 2013). People with primary education or less showed 
Table 4

PARADISE24 mean score comparisons across known-groups based on sociodemographic and clinical background variables in the overall sample $(N=2637)$.

\begin{tabular}{llll}
\hline & M (SD) & p & Effect size \\
\hline Gender & & 0.004 & 0.11 \\
$\quad$ Females & $1.48(0.81)$ & & \\
$\quad$ Males & $1.39(0.79)$ & & \\
Age group & & $<0.001$ & 0.14 \\
$\quad<=30$ & $1.57(0.84)$ & & \\
$31-50$ & $1.42(0.78)$ & & \\
$50+$ & $1.25(0.74)$ & & \\
Education & & $<0.001$ & 0.15 \\
$\quad$ Lower than secondary education & $1.48(0.81)$ & & \\
$\quad$ Secondary education completed or higher & $1.36(0.78)$ & & \\
Clinical setting & & $<0.001$ & 0.86 \\
$\quad$ Inpatients & $1.72(0.74)$ & & \\
$\quad$ Outpatients & $1.09(0.72)$ & & \multirow{2}{*}{0.36} \\
Type of substance & & $<0.001$ & 0.36 \\
$\quad$ Only alcohol & $1.27(0.74)$ & & \\
$\quad$ Drugs or multiple substances & $1.55(0.81)$ & & \\
&
\end{tabular}

Note: Cohen's $d$ was used as effect size measure in the comparisons comprising two groups, while Cohen's $f$ was used as effect size measure in the comparisons across more than two groups. Effect sizes were computed for statistically significant differences only.

higher scores (more frequent and/or severe PSDs) than people who had completed at least secondary education (Poudel et al., 2016). Patients with drug use or multiple substance use reported more frequent and/or severe PSDs than those with alcohol use only (Smith and Larson, 2003). Finally, inpatients scored higher in the severity and/or presence of PSDs than outpatients, at both observed and latent level, which is also congruent to previous research evidence (Garg et al., 1999).

This study comprises a large sample of Finnish people with SUD with different clinical and demographic profiles. However, the results should be interpreted considering the following limitations. The application of the PARADISE24 was not uniformly performed at a specific point in treatment (e.g., in the beginning or the end of treatment). Still, the aim of this study was not to evaluate treatment efficiency. In addition, the study was cross-sectional, so longitudinal invariance analyses and sensitivity to change could not be assessed. Further longitudinal studies concerning clinical utility of the PARADISE24 are also needed in the future. Considering the evidence of unidimensionality of the questionnaire, future research could explore the possibility of creating a reduced version of the PARADISE24. However, the questionnaire was originally created to measure PSDs that are experienced in common across mental, neurological, and substance use disorders. The selection of the items was based on different sources of information including expert opinions and patient experiences (Cieza et al., 2015b). Therefore, content validity of the items across all these disorders should be considered as further studies create a reduced version.

To conclude, this study has showed that the PARADISE24 is a valid tool with high internal consistency in both outpatients and inpatients with substance use disorders. The PARADISE24 is a short but comprehensive tool to collect relevant psychosocial information in people with SUDs in different clinical settings. The PARADISE24 was also able to discriminate between inpatients and outpatients in terms of presence and severity of PSDs. Further longitudinal studies should report the ability of PARADISE24 to detect response to treatment in people with SUDs.

\section{Author disclosures}

\section{Role of funding source}

The research has received funding from the Finnish Foundation for Alcohol Studies. The present study was supported by the Instituto de
Salud Carlos III, Centro de Investigación Biomédica en Red de Salud Mental (CIBERSAM). Javier de la Fuente's work is supported by the FPU predoctoral grant (FPU16/03276) from the Spanish Ministry of Education, Culture and Sports.

\section{Contributors}

TP conceived the research, collected and managed the data, and coordinated the whole process. MC searched the literature, JF designed and performed the statistical analyses under the supervision of FFC. JF and MC wrote the manuscript. FFC, JLAM, JL and TP made critical revisions to the study design and manuscript. All the authors made a substantial contribution to the manuscript and approved its final version.

\section{Conflict of interest}

None declared.

\section{References}

Üstün, T.B., 2010. Measuring Health and Disability: Manual for WHO Disability Assessment Schedule WHODAS 2.0.

Black, R.A., McCaffrey, S., Butler, S.F., 2017. A computerized adaptive testing version of the ASI. Drug Alcohol Depend. 171, e20-e21.

Blanco, C., Iza, M., Rodríguez-Fernández, J.M., Baca-García, E., Wang, S., Olfson, M., 2015. Probability and predictors of treatment-seeking for substance use disorders in the U.S. Drug Alcohol Depend. 149, 136-144.

Caballero, F., Soulis, G., Engchuan, W., Sánchez-Niubó, A., Arndt, H., Ayuso-Mateos, J.L., Haro, J.M., Chatterji, S., Panagiotakos, D.B., 2017. Advanced analytical methodologies for measuring healthy ageing and its determinants, using factor analysis and machine learning techniques: the ATHLOS project. Sci. Rep. 7, 43955.

Cheung, G.W., Rensvold, R.B., 2002. Evaluating goodness-of-fit indexes for testing measurement invariance. Struct. Equ. Model. 9, 233-255.

Cieza, A., Anczewska, M., Ayuso-Mateos, J.L., Baker, M., Bickenbach, J., Chatterji, S., Hartley, S., Leonardi, M., Pitkänen, T., Coenen, M., Gall, H., Kollerits, B., Sabariego, C., Cabello, M., Mellor, B., Vigil, J., Cerniauskaite, M., Covelli, V., Giovannetti, A., Quintas, R., Raggi, A., Schiavolin, S., Ballert, C., Brach, M., Lückenkemper, M., Benbow, A., Hawrot, T., Charzyńska, K., Chrostek, A.,-witaj, P., Roszczyńska-Michta, J., Waszkiewicz, J., Finocchiaro, C., Cogoni, S., Holopainen, A., Jokela, K., Kaskela, T., Levola, J., Tourunen, J., 2015a. Understanding the impact of brain disorders: towards a horizontal epidemiology of psychosocial difficulties and their determinants. PLoS One 10.

Cieza, A., Sabariego, C., Anczewska, M., Ballert, C., Bickenbach, J., Cabello, M., Giovannetti, A., Kaskela, T., Mellor, B., Pitkänen, T., Quintas, R., Raggi, A.,"witaj, P., Chatterji, S., Coenen, M., Gall, H., Kollerits, B., Ayuso-Mateos, J.L., Vigil, J., Leonardi, M., Cerniauskaite, M., Covelli, V., Schiavolin, S., Brach, M., Lückenkemper, M., Baker, M., Benbow, A., Hawrot, T., Hartley, S., Charzyńska, K., Chrostek, A., Roszczyńska-Michta, J., Waszkiewicz, J., Finocchiaro, C., Cogoni, S., Holopainen, A., Jokela, K., Levola, J., Tourunen, J., 2015b. PARADISE 24: a measure to assess the impact of brain disorders on people's lives. PLoS One 10.

Cohen, J., 1988. Statistical Power Analysis for the Behavioral Sciences. Lawrence Erlbaum Associates, New York.

Davidson, M., 2014. Known-Groups Validity. Encyclopedia of Quality of Life and WellBeing Research. Springer, New York.

Deering, D., Robinson, G., Wheeler, A., Pulford, J., Frampton, C., Dunbar, L., Black, S., 2009. Preliminary work towards validating a draft outcome measure for use in the alcohol and drug sector. Te Nui, Pou o te Whakaaro. The National Centre of Mental Health Research, Auckland.

Department of Health, 2007. Drug Misuse and Dependence: UK Guidelines on Clinical Management. DH Publications, London.

Dutra, L., Stathopoulou, G., Basden, S.L., Leyro, T.M., Powers, M.B., Otto, M.W., 2008. A meta-analytic review of psychosocial interventions for substance use disorders. Am. J. Psychiatry 165, 179-187.

García Álvarez, L., Aylin, P., Tian, J., King, C., Catchpole, M., Hassall, S., Whittaker-Axon, K., Holmes, A., 2011. Data linkage between existing healthcare databases to support hospital epidemiology. J. Hosp. Infect. 79, 231-235.

Garg, N., Yates, W.R., Jones, R., Zhou, M., Williams, S., 1999. Effect of gender, treatment site and psychiatric comorbidity on quality of life outcome in substance dependence. Am. J. Addict. 8, 44-54.

Gregorich, Steven E., 2006. Do self-report instruments allow meaningful comparisons across diverse population groups? Testing measurement invariance using the confirmatory factor analysis framework. Med. Care 44 (Suppl 3), S78.

Gsellhofer, B., Küfner, H., Kokkevi, A., Uchtenhagen, A., 1995. European Addiction Severity Index (EuropASI), COST A6. A Guide to Training and Administering EuropASI Interviews. EuropASI, Brussels.

Horn, J.L., 1965. A rationale and test for the number of factors in factor analysis. Psychometrika 30, 179-185.

Hu, L.T., Bentler, P.M., 1999. Cutoff criteria for fit indexes in covariance structure 
analysis: conventional criteria versus new alternatives. Struct. Equ. Model. 6, 1-55.

Levola, J., Kaskela, T., Holopainen, A., Sabariego, C., Tourunen, J., Cieza, A., Pitkänen, T., 2014. Psychosocial difficulties in alcohol dependence: a systematic review of activity limitations and participation restrictions. Disabil. Rehabil. 36, 1227-1239.

Luquiens, A., Reynaud, M., Falissard, B., Aubin, H.J., 2012. Quality of life among alcoholdependent patients: how satisfactory are the available instruments? A systematic review. Drug Alcohol Depend. 125, 192-202.

McLella, A.T., Luborsky, L., Woody, G.E., O’Brien, C.P., 1980. An improved diagnostic evaluation instrument for substance abuse patients: the Addiction Severity Index. J. Nerv. Ment. Dis. 168, 26-33.

Messick, S., 1995. Validity of psychological assessment. Am. Psychol. 50, 741-749.

Moore, T.M., Seavey, A., Ritter, K., McNulty, J.K., Gordon, K.C., Stuart, G.L., 2014 Ecological momentary assessment of the effects of craving and affect on risk for relapse during substance abuse treatment. Psychol. Addict. Behav. 28, 619-624.

Moyer, V.A., 2013. Screening and behavioral counseling interventions in primary care to reduce alcohol misuse: U.S. Preventive Services Task Force recommendation statement. Ann. Intern. Med. 159, 210-218.

Organisation for Economic Co-operation and Development, 2014. Health at a Glance: Europe 2014. Organisation for Economic Co-operation and Development, Paris.

PARADISE24fin, 2018. Retrieved March 28th from http://www.a-klinikka.fi/ paradise24fin.

Pitkänen, T., Levola, J., Tourunen, J., Kaskela, T., Holopainen, A., 2016. Aivotoiminnan häiriöiden yhteydessä yleisesti koetut psykososiaaliset vaikeudet [Common psychosocial difficulties in brain disorders. The Research-Based PARADISE24 Questionnaire]. The Social Insurance Institution of Finland, Helsinki.

Poudel, A., Sharma, C., Gautam, S., Poudel, A., 2016. Psychosocial problems among in dividuals with substance use disorders in drug rehabilitation centers, Nepal. Subst. Abuse Treat. Prev. Policy 11, 28.

Revelle, W., 2008. R Documentation: Procedures for Personality, Psychometric, and Psychological Research. Help pages for package „psych" version, 1-0.

Rossel, Yves, 2012. lavaan: An R Package for Structural Equation Modeling. J. Stat. Softw. 48 (2), 1-36.
Schippers, G.M., Broekman, T.G., Buchholz, A., Koeter, M.W.J., Van Den Brink, W., 2010. Measurements in the Addictions for Triage and Evaluation (MATE): an instrument based on the World Health Organization family of international classifications. Addiction 105, 862-871.

Smith, K.W., Larson, M.J., 2003. Quality of life assessments by adult substance abusers receiving publicly funded treatment in Massachusetts. Am. J. Drug Alcohol Abuse 29, 323-335.

Stevens, L., Verdejo-García, A., Goudriaan, A.E., Roeyers, H., Dom, G., Vanderplasschen, W., 2014. Impulsivity as a vulnerability factor for poor addiction treatment outcomes: a review of neurocognitive findings among individuals with substance use disorders. J. Subst. Abuse Treat. 47, 58-72.

Stucki, G., 2005. International Classification of Functioning, Disability, and Health (ICF): a promising framework and classification for rehabilitation medicine. Am. J. Phys. Med. Rehabil. 84, 733-740.

Twomey, C., Cieza, A., Baldwin, D.S., 2017. Utility of functioning in predicting costs of care for patients with mood and anxiety disorders: a prospective cohort study. Int Clin. Psychopharmacol. 32, 205-212.

Vannieuwenborg, L., Buntinx, F., De Lepeleire, J., 2015. Presenting prevalence and management of psychosocial problems in primary care in Flanders. Arch. Public Health $73,10$.

Velicer, W.F., 1976. Determining the number of components from the matrix of partial correlations. Psychometrika 41, 321-327.

Ware, J.E., 2000. SF-36 health survey update. Spine 25, 3130-3139.

Whiteford, H.A., Degenhardt, L., Rehm, J., Baxter, A.J., Ferrari, A.J., Erskine, H.E., Charlson, F.J., Norman, R.E., Flaxman, A.D., Johns, N., Burstein, R., Murray, C.J.L., Vos, T., 2013. Global burden of disease attributable to mental and substance use disorders: findings from the Global Burden of Disease Study 2010. Lancet 382, 1575-1586.

World Health Organization (WHO), 2001. The International Classification of Functioning, Disability and Health. World Health Organization.

World Health Organization (WHO), 2016. MhGAP Intervention Guide Version 2.0, MhGAP Newsletter. 